\title{
Truncations of Random Unitary Matrices and Young Tableaux
}

\author{
J. Novak \\ Department of Mathematics and Statistics \\ Queen's University, Kingston, Canada \\ jnovak@mast .queensu.ca
}

Submitted: Aug 6, 2006; Accepted: Jan 30, 2007; Published: Feb 7, 2007

Mathematics Subject Classification: 05E10

\begin{abstract}
Let $U$ be a matrix chosen randomly, with respect to Haar measure, from the unitary group $U(d)$. For any $k \leq d$, and any $k \times k$ submatrix $U_{k}$ of $U$, we express the average value of $\left|\operatorname{Tr}\left(U_{k}\right)\right|^{2 n}$ as a sum over partitions of $n$ with at most $k$ rows whose terms count certain standard and semistandard Young tableaux. We combine our formula with a variant of the Colour-Flavour Transformation of lattice gauge theory to give a combinatorial expansion of an interesting family of unitary matrix integrals. In addition, we give a simple combinatorial derivation of the moments of a single entry of a random unitary matrix, and hence deduce that the rescaled entries converge in moments to standard complex Gaussians. Our main tool is the Weingarten function for the unitary group.
\end{abstract}

\section{Introduction and Statement of the Main Theorem}

Consider the unitary group $U(d)$ as a probability space under normalized Haar measure $d U$. Given a random variable $X: U(d) \rightarrow \mathbb{C}$, its expected value is defined to be

$$
\mathbb{E}_{U(d)}(X)=\int_{U(d)} X d U
$$

When studying a random variable $X$, one often wishes to know its moments

$$
\mathbb{E}_{U(d)}\left(X^{m} \bar{X}^{n}\right),
$$

since in many situations the moments of $X$ uniquely determine its distribution. $X$ is called a polynomial random variable if it is polynomial in the entries of $U$, i.e. if there is a polynomial $f \in \mathbb{C}\left[x_{11}, \ldots, x_{d d}\right]$ such that

$$
X(U)=f\left(u_{11}, \ldots, u_{d d}\right)
$$


for all $U \in U(d)$. If $X$ is a polynomial random variable, then

$$
\mathbb{E}_{U(d)}\left(X^{m} \bar{X}^{n}\right)=0 \text { if } m \neq n,
$$

(see [1]), so knowledge of the moments of $X$ reduces to knowledge of the quantities

$$
\mathbb{E}_{U(d)}\left(|X|^{2 n}\right) .
$$

It has recently been shown that certain polynomial random variables on the unitary group encode interesting combinatorial information via their moments. For a random matrix $U \in U(d)$, write its characteristic polynomial as

$$
\operatorname{det}(U-z I)=(-1)^{d} \sum_{j=0}^{d}(-1)^{j} \operatorname{Sc}_{j}(U) z^{d-j} .
$$

$\operatorname{Sc}_{j}(U)$ is called the $j$-th secular coefficient of $U$. In particular,

$$
\begin{aligned}
& \mathrm{Sc}_{1}(U)=\operatorname{Tr}(U) \\
& \mathrm{Sc}_{d}(U)=\operatorname{det}(U) .
\end{aligned}
$$

The combinatorial significance of the secular coefficients of a random Haar-distributed unitary matrix is that their moments enumerate magic squares. It is shown in [2] that for $j n \leq d$,

$$
\mathbb{E}_{U(d)}\left(\left|\mathrm{Sc}_{j}(U)\right|^{2 n}\right)=H_{n}(j),
$$

where $H_{n}(j)$ is the number of $n \times n$ matrices whose entries are nonnegative integers and whose rows and columns all sum to $j$.

For the $S c_{1}(U)=\operatorname{Tr}(U)$, this reduces to the fact that for $n \leq d, \mathbb{E}_{U(d)}\left(|\operatorname{Tr}(U)|^{2 n}\right)=n !$, the number of $n \times n$ permutation matrices. The natural question of what happens when the condition $n \leq d$ is removed was answered by Rains in [6], where it was proved that for any $d$ and $n$

$$
\mathbb{E}_{U(d)}\left(|\operatorname{Tr}(U)|^{2 n}\right)=\sum_{\lambda \vdash n, \ell(\lambda) \leq d}\left(f^{\lambda}\right)^{2},
$$

where the sum is over all partitions $\lambda$ of $n$ and $f^{\lambda}$ denotes the number of Standard Young Tableaux of shape $\lambda$. The significance of this result is that, by the RSK correspondence, $\sum_{\lambda \vdash n, \ell(\lambda) \leq d}\left(f^{\lambda}\right)^{2}$ is the number $f(n, d)$ of permutations in the symmetric group $S_{n}$ having no increasing subsequence of length greater than $d$. Thus Rains's result is equivalent to the remarkable fact that the exponential generating series for $f(n, d)$ has a "closed form" as an integral over the unitary group $U(d)$ :

$$
\sum_{n=0}^{\infty} \frac{f(n, d)}{n !} \frac{z^{n}}{n !}=\int_{U(d)} e^{\sqrt{z} \operatorname{Tr}\left(U+U^{*}\right)} d U .
$$

The elegant method of proof employed in [2], [6] consists of two main ingredients: 
- The secular coefficients of $U$ are the elementary symmetric functions applied to the eigenvalues of $U$.

- The elementary symmetric functions can be written as linear combinations of Schur functions, which are the irreducible characters of the unitary group and thus satisfy orthogonality relations.

In this paper we will study polynomial random variables on $U(d)$ that are not symmetric functions of the eigenvalues. For example, suppose that we are given a random matrix $U \in U(d)$, and we want to calculate the moments of a single entry of $U$

$$
\mathbb{E}_{U(d)}\left(\left|u_{i j}\right|^{2 n}\right)
$$

(note that since the permutation matrices are in $U(d)$ and Haar measure is unitary invariant, all entries of $U$ are equidistributed). More generally, for a positive integer $k$ with $1 \leq k \leq d$, let $U_{k}$ denote the $k \times k$ upper left corner of $U$. We could ask about the moments of the matrix $U_{k}$

$$
\mathbb{E}_{U(d)}\left(\left|\operatorname{Tr}\left(U_{k}\right)\right|^{2 n}\right)
$$

(again, since the permutation matrices are in $U(d)$, the traces of any two $k \times k$ submatrices of $U$ are equidistributed). The methods used in [6], [2] will not work in this situation, since we are no longer dealing with the eigenvalues of a unitary matrix, but rather the eigenvalues of one of its submatrices, so that Schur orthogonality is not directly applicable. However, quite surprisingly, there is a combinatorial formula for these moments that maintains the structure of that for the full matrix case.

Theorem 1. Let $U$ be a matrix chosen randomly with respect to Haar measure from the unitary group $U(d)$, and let $U_{k}$ be its $k \times k$ upper left corner. We have

$$
\mathbb{E}_{U(d)}\left(\left|\operatorname{Tr}\left(U_{k}\right)\right|^{2 n}\right)=\sum_{\lambda \vdash n, \ell(\lambda) \leq k}\left(f^{\lambda}\right)^{2} \frac{s_{\lambda}\left(1^{k}\right)}{s_{\lambda}\left(1^{d}\right)} .
$$

Equivalently, for any $z \in \mathbb{C}$ we have

$$
\int_{U(d)} e^{\sqrt{z} \operatorname{Tr}\left(U_{k}+U_{k}^{*}\right)} d U=\sum_{n=0}^{\infty}\left(\sum_{\lambda \vdash n, \ell(\lambda) \leq k} \frac{\left(f^{\lambda}\right)^{2}}{n !} \frac{s_{\lambda}\left(1^{k}\right)}{s_{\lambda}\left(1^{d}\right)}\right) \frac{z^{n}}{n !} .
$$

Here, for a positive integer $r, s_{\lambda}\left(1^{r}\right)$ denotes the number of Semistandard Young Tableaux on the shape $\lambda$ with entries from the set $[r]=\{1, \ldots, r\}$. The notation $s_{\lambda}\left(1^{r}\right)$ is shorthand for the value of the Schur function $s_{\lambda}$ obtained by setting the first $r$ variables equal to 1 and making the remaining variables 0 .

Note that we recover Rains's result by setting $k=d$ in Theorem 1 . 


\section{Applications of the Main Theorem}

Before proving Theorem 1, we will give two applications. First we use the Colour-Flavour Transformation of lattice gauge theory to give another formulation of Theorem 1, which gives a combinatorial expansion for an interesting type of matrix integral. Then we use Theorem 1 to give simple combinatorial proofs of known probabalistic results regarding the moments and limiting distribution of the individual entries of a random unitary matrix.

The matrix $U_{k}$ is called the $k$ th truncation of $U$. Truncations of random unitary matrices have been the subject of several recent articles (see [3], [5], [8]). Interest in truncations of random unitary matrices stems from their relation to the physics of quantum chaotic scattering [8].

Truncations of random unitary matrices are closely related to the so-called ColourFlavour Transformation (CFT) from physics introduced in [11]. As explained in [10], the CFT trades an integration over a "colour" gauge group for an integration over a certain supersymmetric coset space, or "flavour" space. The physical terminology comes from lattice gauge theory. There are several versions of the CFT, see [3] for details. The one that we will use can be formulated mathematically as follows (see [3], [10]). Let $k \leq d$, and let $X, Y \in \mathbb{C}^{d \times k}$ be constant matrices. Then

$$
\int_{U(d)} e^{\operatorname{Tr}\left(Y^{*} U X+X^{*} U^{*} Y\right)} d U=\int_{U(k)} e^{\operatorname{Tr}\left(X^{*} X V^{*}+V Y^{*} Y\right)} \operatorname{det}\left(V Y^{*} X\right)^{k-d} d V .
$$

This variant of the CFT shows a certain recursive property of integration on the unitary group. In [10], the interesting observation was made that the integral on the left does not change if we replace the unitary group $U(d)$ with the special unitary group $S U(d)$ provided $k<d$ :

$$
\int_{U(d)} e^{\operatorname{Tr}\left(Y^{*} U X+X^{*} U^{*} Y\right)} d U=\int_{S U(d)} e^{\operatorname{Tr}\left(Y^{*} U X+X^{*} U^{*} Y\right)} d U, k<d .
$$

Now let $x$ be a nonnegative real number and take $X(x)=Y(x) \in \mathbb{C}^{d \times k}$ to be the matrix with $x^{\frac{1}{4}}$ along the main diagonal and 0's elsewhere. Then

$$
\begin{aligned}
Y(x)^{*} U X(x) & =\sqrt{x} U_{k} \\
X(x)^{*} U^{*} Y(x) & =\sqrt{x} U_{k}^{*} \\
V Y(x)^{*} X(x) & =\sqrt{x} V .
\end{aligned}
$$

Thus applying the CFT yields

Corollary 2.1. Let $k, d$ be positive integers with $k \leq d$, and let $x$ be a nonnegative real number. Define

$$
f(n, d, k):=\sum_{\lambda \vdash n, \ell(\lambda) \leq k} \frac{\left(f^{\lambda}\right)^{2}}{n !} \frac{s_{\lambda}\left(1^{k}\right)}{s_{\lambda}\left(1^{d}\right)} .
$$


The exponential generating series for $f(n, d, k)$ has two different closed forms, one as an integral over $U(d)$ and one as an integral over $U(k)$ :

$$
\sum_{n=0}^{\infty} f(n, d, k) \frac{x^{n}}{n !}=\int_{U(d)} e^{\sqrt{x} \operatorname{Tr}\left(U_{k}+U_{k}^{*}\right)}=x^{\frac{k(k-d)}{2}} \int_{U(k)} e^{\sqrt{x} \operatorname{Tr}\left(V+V^{*}\right)} \operatorname{det}(V)^{k-d} d V .
$$

Also, in the range $k<d$, we have a third closed form as an integral over the group $S U(d)$ :

$$
\sum_{n=0}^{\infty} f(n, d, k) \frac{x^{n}}{n !}=\int_{S U(d)} e^{\sqrt{x} \operatorname{Tr}\left(U_{k}+U_{k}^{*}\right)} d U
$$

We remarked in the Introduction that the extreme case $k=d$ of Theorem 1 is Rains's result, and is of relevance to the longest increasing subsequence problem. The other extreme $k=1$ is also of interest, since this corresponds to the computation of the moments of a single entry of a random unitary matrix.

Corollary 2.2. In the special case $k=1$, we have

$$
\mathbb{E}_{U(d)}\left(\left|u_{i j}\right|^{2 n}\right)=\frac{n !}{d(d+1) \ldots(d+n-1)}=\left(\begin{array}{c}
d+n-1 \\
n
\end{array}\right)^{-1} .
$$

Proof. For $k=1$ the only contribution to the sum is made by the single partition whose diagram is a row of $n$ boxes. Thus we have

$$
\int_{U(d)}\left|u_{i j}\right|^{2 n} d U=\frac{1}{s_{n}\left(1^{d}\right)} .
$$

The generalized hook length formula asserts that

$$
s_{\lambda}\left(1^{d}\right)=\prod_{\square \in \lambda} \frac{d+c(\square)}{h(\square)},
$$

where $c(\square)$ is the content of the box, and $h(\square)$ is its hook length (see [9]). For the single row partition of $n$, this gives

$$
s_{n}\left(1^{d}\right)=\frac{d(d+1) \ldots(d+n-1)}{n !},
$$

and the result follows.

Explicitly knowing the moments of $u_{i j}$ makes it easy to determine its limiting distribution. Recall that if $x, y$ are Gaussian random variables with mean 0 and variance $1 / 2$, then the random variable $z=x+i y$ is called a standard complex Gaussian.

Corollary 2.3. As $d \rightarrow \infty$, the random variable $\sqrt{d} u_{i j}$ converges in moments to a standard complex Gaussian random variable. 
Proof. It is well-known (see for instance [5]) that the moments of a standard complex Gaussian $z$ are given by

$$
\mathbb{E}\left(z^{m} \bar{z}^{n}\right)=\delta_{m n} n !
$$

Corollary 1.2 shows that

$$
\begin{aligned}
\mathbb{E}_{U(d)}\left(\left(\sqrt{d} u_{i j}\right)^{m}\left(\sqrt{d} \bar{u}_{i j}\right)^{n}\right) & =\delta_{m n} \frac{d^{n} n !}{d(d+1) \ldots(d+n-1)} \\
& =\delta_{m n} \frac{n !}{(1)\left(1+\frac{1}{d}\right) \ldots\left(1+\frac{n-1}{d}\right)} \\
& \rightarrow \delta_{m n} n ! .
\end{aligned}
$$

\section{The Weingarten Function}

In order to prove Theorem 1, one needs to connect unitary expectations to symmetric function theory by some method other than applying symmetric functions to eigenvalues. This can be done using the Weingarten function introduced in [1], which is a powerful tool for computing the moments of polynomial random variables on the unitary group. The Weingarten function has already been used in free probability theory to prove asymptotic freeness results for random unitary matrices (see the recent book [4] for a clear account of this).

For any positive integers $d$ and $n$, define a function $\operatorname{Wg}(d, n, \cdot): S_{n} \rightarrow \mathbb{Q}$ by

$$
\mathrm{Wg}(d, n, \sigma):=\frac{1}{n !^{2}} \sum_{\lambda \vdash n, \ell(\lambda) \leq d} \frac{\left(f^{\lambda}\right)^{2}}{s_{\lambda}\left(1^{d}\right)} \chi^{\lambda}(\sigma),
$$

where $\chi^{\lambda}$ is the irreducible character of $S_{n}$ labelled by $\lambda$.

The following integration formula was proved in [1].

Theorem 2. Let $i, j, i^{\prime}, j^{\prime}:[n] \rightarrow[d]$ be any functions. Then

$$
\begin{aligned}
& \int_{U(d)} u_{i(1) j(1)} \ldots u_{i(n) j(n)} \bar{u}_{i^{\prime}(1) j^{\prime}(1)} \ldots \bar{u}_{i^{\prime}(n) j^{\prime}(n)} d U \\
& =\sum_{\sigma, \tau \in S_{n}} \delta_{i(1) i^{\prime}(\sigma(1))} \ldots \delta_{i(n) i^{\prime}(\sigma(n))} \delta_{j(1) j^{\prime}(\tau(1))} \ldots \delta_{j(n) j^{\prime}(\tau(n))} \operatorname{Wg}\left(d, n, \tau \sigma^{-1}\right),
\end{aligned}
$$

where $\delta$ is the Kronecker delta.

We can succinctly express the moments of $\operatorname{Tr}\left(U_{k}\right)$ in terms of the Weingarten function as follows: 
Lemma 1. For any positive integers $n$ and $k$, where $1 \leq k \leq d$, we have

$$
\mathbb{E}_{U(d)}\left(\left|\operatorname{Tr}\left(U_{k}\right)\right|^{2 n}\right)=n ! \sum_{\alpha}\left(\begin{array}{l}
n \\
\alpha
\end{array}\right) \sum_{\sigma \in S_{\alpha}} \operatorname{Wg}(d, n, \sigma),
$$

where the outer sum runs over all weak $k$-part compositions $\alpha$ of $n$, and the inner sum runs over all permutations in the Young subgroup $S_{\alpha}$ of $S_{n}$.

Proof. This is really just a calculation. We expand

$$
\begin{aligned}
\left|\operatorname{Tr}\left(U_{k}\right)\right|^{2 n} & =\left|u_{11}+\cdots+u_{k k}\right|^{2 n} \\
& =\left(u_{11}+\cdots+u_{k k}\right)^{n}\left(\bar{u}_{11}+\cdots+\bar{u}_{k k}\right)^{n} \\
& =\sum_{\alpha} \sum_{\beta}\left(\begin{array}{l}
n \\
\alpha
\end{array}\right)\left(\begin{array}{l}
n \\
\beta
\end{array}\right) u^{\alpha} \bar{u}^{\beta},
\end{aligned}
$$

where we are summing over all pairs of weak $k$-part compositions of $n, \alpha=\left(a_{1}, \ldots, a_{k}\right)$ and $\beta=\left(b_{1}, \ldots, b_{k}\right)$. We are using multi-index notation,

$$
\begin{aligned}
\left(\begin{array}{l}
n \\
\alpha
\end{array}\right) & =\left(\begin{array}{c}
n \\
a_{1}, \ldots, a_{k}
\end{array}\right) \\
u^{\alpha} & =u_{11}^{a_{1}} \ldots u_{k k}^{a_{k}} \\
\left(\begin{array}{c}
n \\
\beta
\end{array}\right) & =\left(\begin{array}{c}
n \\
b_{1}, \ldots, b_{k}
\end{array}\right) \\
\bar{u}^{\beta} & =\bar{u}_{11}^{b_{1}} \ldots \bar{u}_{k k}^{b_{k}}
\end{aligned}
$$

Hence

$$
\mathbb{E}_{U(d)}\left(\left|\operatorname{Tr}\left(U_{k}\right)\right|^{2 n}\right)=\sum_{\alpha} \sum_{\beta}\left(\begin{array}{l}
n \\
\alpha
\end{array}\right)\left(\begin{array}{l}
n \\
\beta
\end{array}\right) \mathbb{E}_{U(d)}\left(u^{\alpha} \bar{u}^{\beta}\right) .
$$

We will use the Weingarten integration formula to evaluate the expectation $\mathbb{E}_{U(d)}\left(u^{\alpha} \bar{u}^{\beta}\right)$ for a fixed pair of compositions $\alpha, \beta$. Implicitly define coordinate functions $i_{\alpha}, j_{\alpha}, i_{\beta}, j_{\beta}$ : $[n] \rightarrow[d]$ by setting

$$
\begin{aligned}
& \int_{U(d)} u_{i_{\alpha}(1) j_{\alpha}(1)} \ldots u_{i_{\alpha}(n) j_{\alpha}(n)} \bar{u}_{i_{\beta}(1) j_{\beta}(1)} \ldots \bar{u}_{i_{\beta}(n) j_{\beta}(n)} d U \\
& :=\int_{U(d)} u_{11}^{a_{1}} \ldots u_{k k}^{a_{k}} \bar{u}_{11}^{b_{1}} \ldots \bar{u}_{k k}^{b_{k}} d U \\
& =\mathbb{E}_{U(d)}\left(u^{\alpha} \bar{u}^{\beta}\right) .
\end{aligned}
$$

Applying the Weingarten integration formula, we have

$$
\begin{aligned}
& \mathbb{E}_{U(d)}\left(u^{\alpha} \bar{u}^{\beta}\right) \\
& =\sum_{\sigma, \tau \in S_{n}} \delta_{i_{\alpha}(1) i_{\beta}(\sigma(1))} \ldots \delta_{i_{\alpha}(n) i_{\beta}(\sigma(n))} \delta_{j_{\alpha}(1) j_{\beta}(\tau(1))} \ldots \delta_{j_{\alpha}(n) j_{\beta}(\tau(n))} \mathrm{Wg}\left(d, n, \tau \sigma^{-1}\right) .
\end{aligned}
$$


Since we are only taking entries from the diagonal, we have $i_{\alpha}=j_{\alpha}$ and $i_{\beta}=j_{\beta}$. Moreover, the level sets of these functions are easy to read off:

$$
\begin{aligned}
i_{\alpha}^{-1}(1) & =\left[1, a_{1}\right] \\
i_{\alpha}^{-1}(2) & =\left[a_{1}+1, a_{1}+a_{2}\right] \\
\vdots & \\
i_{\alpha}^{-1}(k) & =\left[n-a_{k}+1, n\right]
\end{aligned}
$$

and

$$
\begin{aligned}
i_{\beta}^{-1}(1) & =\left[1, b_{1}\right] \\
i_{\beta}^{-1}(2) & =\left[b_{1}+1, b_{1}+b_{2}\right] \\
\vdots & \\
i_{\beta}^{-1}(k) & =\left[n-b_{k}+1, n\right]
\end{aligned}
$$

Hence

$$
\begin{aligned}
\prod_{r=1}^{a_{1}} \delta_{i_{\alpha}(r) i_{\beta}(\sigma(r))} & =\prod_{r=1}^{a_{1}} \delta_{1 i_{\beta}(\sigma(r))} \\
\prod_{r=a_{1}+1}^{a_{1}+a_{2}} \delta_{i_{\alpha}(r) i_{\beta}(\sigma(r))} & =\prod_{r=a_{1}+1}^{a_{1}+a_{2}} \delta_{2 i_{\beta}(\sigma(r))} \\
\vdots & \\
\prod_{r=n-a_{k}+1}^{n} \delta_{i_{\alpha}(r) i_{\beta}(\sigma(r))} & =\prod_{r=n-a_{k}+1}^{n} \delta_{k i_{\beta}(\sigma(r))},
\end{aligned}
$$

Thus in order for the product

$$
\delta_{i_{\alpha}(1) i_{\beta}(\sigma(1))} \ldots \delta_{i_{\alpha}(n) i_{\beta}(\sigma(n))}
$$

to be nonzero, we see that $\sigma$ must bijectively map the interval $\left[1, a_{1}\right]$ onto the interval $\left[1, b_{1}\right]$, and $\sigma$ must also bijectively map the interval $\left[a_{1}+1, a_{1}+a_{2}\right]$ onto the interval $\left[b_{1}+1, b_{1}+b_{2}\right]$, etc. Similarly, in order for the product

$$
\delta_{j_{\alpha}(1) j_{\beta}(\tau(1))} \ldots \delta_{j_{\alpha}(n) j_{\beta}(\tau(n))}
$$

to be nonzero, we see that $\tau$ must bijectively map the interval $\left[1, a_{1}\right]$ onto the interval $\left[1, b_{1}\right]$, and $\tau$ must also bijectively map the interval $\left[a_{1}+1, a_{1}+a_{2}\right]$ onto the interval $\left[b_{1}+1, b_{1}+b_{2}\right]$, etc. Thus we see that the expectation $\mathbb{E}_{U(d)}\left(u^{\alpha} \bar{u}^{\beta}\right)$ is zero unless:

- $\alpha=\beta$, i.e. these two are the same weak $k$-part composition of $n$, 
- $\sigma, \tau$ are both in the Young subgroup $S_{\alpha}$, i.e. the subgroup of permutations in $S_{n}$ that permute the first $a_{1}$ symbols amongst themselves, the next $a_{2}$ symbols amongst themselves, etc.

Thus

$$
\begin{aligned}
\mathbb{E}_{U(d)}\left(\left|\operatorname{Tr}\left(U_{k}\right)\right|^{2 n}\right) & =\sum_{\alpha}\left(\begin{array}{l}
n \\
\alpha
\end{array}\right)^{2} \mathbb{E}_{U(d)}\left(u^{\alpha} \bar{u}^{\alpha}\right) \\
& =\sum_{\alpha}\left(\begin{array}{l}
n \\
\alpha
\end{array}\right)^{2} \sum_{\sigma, \tau \in S_{\alpha}} \operatorname{Wg}\left(d, n, \tau \sigma^{-1}\right) \\
& =\sum_{\alpha}\left(\begin{array}{l}
n \\
\alpha
\end{array}\right)^{2} \alpha ! \sum_{\sigma \in S_{\alpha}} \operatorname{Wg}(d, n, \sigma) \\
& =n ! \sum_{\alpha}\left(\begin{array}{l}
n \\
\alpha
\end{array}\right) \sum_{\sigma \in S_{\alpha}} \operatorname{Wg}(d, n, \sigma)
\end{aligned}
$$

\section{Proof of the Main Theorem}

We are now in a position to prove Theorem 1.

Proof. We work with the sum on the right hand side of Lemma 1. Plugging in the definition of the Weingarten function, we have

$$
n ! \sum_{\alpha}\left(\begin{array}{l}
n \\
\alpha
\end{array}\right) \sum_{\sigma \in S_{\alpha}} \operatorname{Wg}(d, n, \sigma)=n ! \sum_{\alpha}\left(\begin{array}{l}
n \\
\alpha
\end{array}\right) \sum_{\sigma \in S_{\alpha}} \frac{1}{n !^{2}} \sum_{\lambda \vdash n, \ell(\lambda) \leq d} \frac{\left(f^{\lambda}\right)^{2}}{s_{\lambda}\left(1^{d}\right)} \chi^{\lambda}(\sigma) .
$$

Changing order of summation, this becomes

$$
\sum_{\lambda \vdash n, \ell(\lambda) \leq d}\left(f^{\lambda}\right)^{2} \frac{1}{s_{\lambda}\left(1^{d}\right)} \sum_{\alpha} \frac{1}{\alpha !} \sum_{\sigma \in S_{\alpha}} \chi^{\lambda}(\sigma)=\sum_{\lambda \vdash n, \ell(\lambda) \leq d}\left(f^{\lambda}\right)^{2} \frac{1}{s_{\lambda}\left(1^{d}\right)} \sum_{\alpha}\left\langle 1, \chi^{\lambda}\right\rangle_{S_{\alpha}},
$$

where the inner product $\langle\cdot, \cdot\rangle_{S_{\alpha}}$ is the averaged dot product on the space $C F\left(S_{\alpha}\right)$ of complex-valued class functions on the group $S_{\alpha}$. Note that this sum may be written as

$$
\sum_{\lambda \vdash n, \ell(\lambda) \leq d}\left(f^{\lambda}\right)^{2} \frac{1}{s_{\lambda}\left(1^{d}\right)} \sum_{\alpha}\left\langle 1, \chi^{\lambda} \downarrow_{S_{\alpha}}^{S_{n}}\right\rangle_{S_{\alpha}},
$$

where $\chi^{\lambda} \downarrow_{S_{\alpha}}^{S_{n}}$ is the restriction of the irreducible character $\chi^{\lambda}$ of $S_{n}$ to the subgroup $S_{\alpha}$. Now, the function which is identically 1 is the character of the trivial representation of $S_{\alpha}$. Thus we may apply Frobenius reciprocity (see for instance [7]);

$$
\left\langle 1, \chi^{\lambda} \downarrow_{S_{\alpha}}^{S_{n}}\right\rangle_{S_{\alpha}}=\left\langle 1 \uparrow_{S_{\alpha}}^{S_{n}}, \chi^{\lambda}\right\rangle_{S_{n}}
$$


where $1 \uparrow S_{S_{\alpha}}^{S_{n}}$ is the induction of the trivial character of $S_{\alpha}$ to $S_{n}$.

The final step in the proof relies on the characteristic map $\operatorname{ch}_{n}: C F\left(S_{n}\right) \rightarrow \Lambda^{n}$, where $\Lambda^{n}$ is the inner product space of degree $n$ symmetric functions equipped with the Hall inner product $\langle\cdot, \cdot\rangle_{\Lambda^{n}}$ (see [7] or [9]). The characteristic function is an isometry, and has the following important properties:

$$
\begin{aligned}
\operatorname{ch}_{n}\left(1 \uparrow{ }_{S_{\alpha}}^{S_{n}}\right) & =h_{\alpha} \\
\operatorname{ch}_{n}\left(\chi^{\lambda}\right) & =s_{\lambda},
\end{aligned}
$$

where $h_{\alpha}$ is the complete homogeneous symmetric function indexed by $\alpha$, and $s_{\lambda}$ is the Schur function indexed by $\lambda$. Thus we have

$$
\left\langle 1 \uparrow_{S_{\alpha}}^{S_{n}}, \chi^{\lambda}\right\rangle_{S_{n}}=\left\langle\operatorname{ch}_{n}\left(1 \uparrow_{S_{\alpha}}^{S_{n}}\right), \operatorname{ch}_{n}\left(\chi^{\lambda}\right)\right\rangle_{S_{n}}=\left\langle h_{\alpha}, s_{\lambda}\right\rangle_{\Lambda^{n}}
$$

It is well-known that the Schur functions constitute an orthonormal basis for $\Lambda^{n}$, and that the coordinates of the complete homogeneous symmetric functions with respect to the basis of Schur functions are the Kostka numbers (see [9]). That is,

$$
h_{\alpha}=\sum_{\mu \vdash n} K_{\mu \alpha} s_{\mu}
$$

where the Kostka number $K_{\mu \alpha}$ is by definition the number of semistandard Young tableaux on the diagram of $\mu$ with content vector $\alpha$. Thus,

$$
\left\langle h_{\alpha}, s_{\lambda}\right\rangle_{\Lambda^{n}}=\sum_{\mu \vdash n} K_{\mu \alpha}\left\langle s_{\mu}, s_{\lambda}\right\rangle_{\Lambda^{n}}=K_{\lambda, \alpha} .
$$

Thus we have

$$
\begin{aligned}
\sum_{\lambda \vdash n, \ell(\lambda) \leq d}\left(f^{\lambda}\right)^{2} \frac{1}{s_{\lambda}\left(1^{d}\right)} \sum_{\alpha}\left\langle 1, \chi^{\lambda}\right\rangle_{S_{\alpha}} & =\sum_{\lambda \vdash n, \ell(\lambda) \leq d}\left(f^{\lambda}\right)^{2} \frac{1}{s_{\lambda}\left(1^{d}\right)} \sum_{\alpha} K_{\lambda \alpha} \\
& =\sum_{\lambda \vdash n, \ell(\lambda) \leq d}\left(f^{\lambda}\right)^{2} \frac{s_{\lambda}\left(1^{k}\right)}{s_{\lambda}\left(1^{d}\right)},
\end{aligned}
$$

where the last equality follows from the fact that, by definition,

$$
\sum_{\alpha} K_{\lambda \alpha}=s_{\lambda}\left(1^{k}\right)
$$

since the sum runs over all weak $k$-part compositions of $n$.

Finally, we remark that if $\lambda$ is a partition of $n$ with $\ell(\lambda)>k$, then $s_{\lambda}\left(1^{k}\right)=0$. Hence,

$$
\sum_{\lambda \vdash n, \ell(\lambda) \leq d}\left(f^{\lambda}\right)^{2} \frac{s_{\lambda}\left(1^{k}\right)}{s_{\lambda}\left(1^{d}\right)}=\sum_{\lambda \vdash n, \ell(\lambda) \leq k}\left(f^{\lambda}\right)^{2} \frac{s_{\lambda}\left(1^{k}\right)}{s_{\lambda}\left(1^{d}\right)},
$$

which proves our theorem. 


\section{Conclusion}

The results in this paper arose from the author's attempts to evaluate known unitary integrals via the Weingarten function. It was observed that with the Weingarten method one gets information about related integrals where the matrices in the integrand are truncated with no extra work.

The notion of Weingarten function has been defined for the orthogonal and symplectic groups $O(d)$ and $S p(d)$, and similar expansion formulas have been proved for expectations of polynomial random variables on these groups [1]. There are also versions of the ColourFlavour transformation for the orthogonal and symplectic groups (indeed, the ColourFlavour transformations are all intimately related to Howe duality, see [12]). Thus there are likely analogues of Theorem 1 for truncations of random orthogonal and symplectic matrices.

\section{Acknowledgements}

I am grateful to Roland Speicher and Jamie Mingo for helpful discussions, and to Richard Stanley for encouraging comments.

\section{References}

[1] B. Collins and P. Śniady, Integration with respect to the Haar measure on unitary, orthogonal, and symplectic groups, Commun. Math. Phys. 264 (2006), 773-795.

[2] P. Diaconis and A. Gamburd, Random matrices, magic squares and matching polynomials, Electron. J. Combinatorics 11(2), (2004), R2.

[3] Y. Fyodorov and B. Khoruzhenko, A few remarks on Colour-Flavour transformations, truncations of random unitary matrices, Berezin reproducing kernels and Selberg type integrals. math-ph/0610045

[4] A. Nica and R. Speicher, Lectures on the Combinatorics of Free Probability, London Mathematical Society Lecture Notes Series, No. 335

[5] D. Petz and J. Reffy, On asymptotics of large Haar distributed unitary matrices. math.PR/0310338

[6] E.M. Rains, Increasing subsequences and the classical groups, Electron. J. Combinatorics 5 (1998), R12.

[7] B. Sagan, The Symmetric Group: Representations, Combinatorial Algorithms, and Symmetric Functions, Springer Graduate Texts in Mathematics 203.

[8] H.-J. Sommers and K. Zyczkowski, Truncations of random unitary matrices, J. Phys. A: Math. Gen. 33 (2002), 2045-2057

[9] R. Stanley, Enumerative Combinatorics, Volume 2, Cambridge University Press, Cambridge, 1999. 
[10] Y. Wei and T. Wettig, Bosonic color-flavor transformation for the special unitary group, J. Math. Phys. 46, Art. No. 072306, 2006.

[11] M.R. Zirnbauer, The Color-Flavor Transformation and a new approach to quantum chaotic maps. Proceedings of the XIIth International Congress of Mathematical Physics, Brisbane 13-19 July 1997, ed. D. De Wit, A.J. Braken, M.D. Gould, P.A. Pearce (International Press Inc. Cambridge, MA) pp.290-297, 1999

[12] M.R. Zirnbauer, Howe Duality and the Color-Flavor Transformation, talk at: New Directions in Nonperturbative QCD, Trento 27-31 March 2006, available at http://www.ect.it/Meetings/ConfsWksAndCollMeetings/ConfWksDocument/ 2006/Talks/27-31March/zirnbauer.pdf 